https://doi.org/10.47370/2078-1024-2021-13-4-130-137

УДК 378.018:17

Губжокова Н.К.

МОРАЛЬНО-ЭТИЧЕСКИЙ АСПЕКТ ДИСТАНЦИОННОГО ОБРАЗОВАНИЯ

\author{
Губжокова Нуриет Карпушевна, \\ кандидат филологических наук, дочент кафедры философии, сочиологии \\ и педагогики ФГБОУ ВО «Майкопский государственный технологический \\ университет», Майкоп, Россия, \\ e-mail: 'gubzhockova.nuriet2015@yandex.ru, \\ тел.: +7.(928) 4696452
}

\title{
Аннотация
}

Дистанционное обучение давно уже является одним из интегральных трендов развития современной образовательной системы, представляя собой совокупность новых способов передачи информации инновационных методов взаимодействия педагогов и учащихся. Дистанционное образование, появившееся как способ предоставления равных возможностей всем потенциальным участникам образовательного процесса, в свою очередь является источником новых проблем, чем и предопределена актуальность данной работы. Целью настоящей работы является выявление важнейших проблем морально-этического плана, в том числе и тех, что обострились в период пандемии COVID-19, ставшей серьезным вызовом для мировой образовательной системы. В исследовании использовались методы анализа, аналогии, обобщения. В работе востребован и опыт дистанционной работы преподавателей МГТУ в условиях пандемии.

Результаты: в статье выделены основные угрозы этического характера, связанные с онлайн-образованием, которые распределены по характеру и степени воздействия на процесс обучения.

Ключевые выводы: морально-этический аспект дистанционного образования является одним из важнейших при постепенном переходе на новый формат обучения. Особую остроту эти проблемы приобрели в ситуации пандемии COVID-19, в ходе которой было осуществлено вынужденное и ускоренное внедрение дистанционных форм обучения в педагогическую практику, что выявило уязвимость целых регионов и категорий населения, для которых онлайн-формат обучения оказался крайне затрудненным. Опыт работы системы образования в условиях пандемии требует глубокого изучения и обобщения для корректирования методов и темпов инновационных процессов в современной педагогике, а также для уточнения места, характера и значимости дистанционного обучения в образовательной системе.

Ключевые слова: дистанционное образование, обучение, онлайн, цифровая этика, пандемия, COVID-19

Для цитирования: Тубжокова Н.К. Морально-этический аспект дистанионного образования // Вестиик Майкопского государственного технологического универсиmema. 2021. Tом 13, № 4. C. 130-137. https:/doi.org/10.47370/2078-1024-2021-13-4-130-137. 


\title{
Gubzhokova N.K.
}

\section{MORAL AND ETHICAL ASPECT OF DISTANCE EDUCATION}

\author{
Gubzhokova Nuriet Karpushevna, \\ Candidate of Philology, an Associate Professor of the Department of Philoso- \\ phy, Sociology andiPedagogy of FSBEI HE "Maykop State Technological Uni- \\ versity", Maykop, Russia, \\ e-mail: gubzhockova.nuriet2015@yandex.ru, \\ tel.: +7.(928) 4696452
}

\section{Annotation}

Distance learning has been one of the integral trends in the development of the modern educational system, representing a set of new ways of transferring information, innovative methods of interaction between teachers and students. Distance education, which has emerged as a way of providing equal opportunities to all potential participants in the educational process, is a source of new problems, which predetermines the relevance of the research. The purpose of the research is to identify the most important moral and ethical problems, including those that have worsened during the COVID-19 pandemic, which has become a serious challenge for the global educational system. The research methods used are analysis, analogy, generalization. The experience of remote work of MSTU teachers in the pandemic period is also in demand.

The results: the article highlights the main ethical threats associated with online education, which are distributed according to the nature and degree of impact on the learning process.

The key findings: the moral and ethical aspect of distance education is one of the most important in the gradual transition to a new teaching format. These problems became especially acute in the situation of the COVID-19 pandemic, during which the forced and accelerated introduction of distance learning into pedagogical practice has been carried out, which revealed the vulnerability of entire regions and categories of the population. The experience of the education system in the pandemic requires deep study and generalization to adjust the methods and rates of innovative processes in modern Pedagogy, as well as to clarify the place, nature and importance of distance learning in the educational system.

Keywords: distance education, learning, online, digital ethics, pandemic, COVID-19

For citation: Gubzhokova N.K. Moral and ethical aspect of distance education // Vestnik Majkopskogo gosudarstvennogo tehnologičeskogo universiteta. 2021. Volume 13, No. 4. P. 130-137. https://oi.org/10.47370/2078-1024-2021-13-4-130-137.

В последние десятилетия дистанционное обучение постепенно становится неотъемлемой составной частью системы образования. Ныне оно уже переросло рамки второстепенного дополнения к традиционному образовательному процессу, неизбежно приводя к появлению принципиально иных способов передачи информации от обучающих к обучаемым инновационных методов взаимодействия педагогов и учащихся [1, с. 71$]$.
Подобный пересмотр самой педагогической парадигмы порождает массу проблем самого разного свойства, и, возможно, этические проблемы являются самыми острыми из них.

Особую остроту многие из этих проблем приобрели в ситуации пандемии COVID-19, кардинально изменившей облик современного мира и вызвавшей глубокие трансформации в сфере образования (и в том числе, вынужденное 
и ускоренное внедрение дистанционных форм обучения в педагогическую практику).

Часть этих проблем и связанных с ними моральных коллизий и даже потенциальных угроз - это реалии сегодняшнего дня. Мы с ними сталкиваемся в повседневной практике дистанционного образования. О существовании других мы знаем из научных статей и аналитических обзоров; они могут стать актуальными в ближайшее время, по мере дальнейшей цифровизации общества в целом, и высшего образования в частности. К настоящему времени сформировалась целая отрасль этического знания - информационная (цифровая) этика, в том числе занимающаяся и проблемами современного цифрового образования $[1$, с. 71$]$.

Эти проблемы могут носить как глобальный, так и локальный характер.

В связи с этим, важным представляется изучение морально-этического аспекта дистанционного образования. При этом можно выделить ряд задач, заслуживающих рассмотрения в процессе исследования:

- выявление основных проблем дистанционного образования, имеющих морально-этический характер;

- акцентирование внимания на проблемах, которые обострились в период пандемии COVID-19, ставшей серьезным вызовом для мировой образовательной системы;

- распределение угрозы моральноэтического плана по характеру и степени воздействия на процесс обучения (глобальный или местный, актуальные уже в настоящее время или имеющие гипотетические последствия).

Следует отметить, что само появление онлайн-образования отнюдь не было связано с какими-то эпидемическими процессами и карантинными мероприятиями. Бурное развитие дистанта во многом было продиктовано этической проблемой неравных возможностей участников образовательного процесса. Потенциальные студенты в зависимости от гражданства, периферийного характера места проживания, материального статуса, а зачастую - от расовой и гендерной принадлежности изначально имели неодинаковые шансы получения высшего образования - причем не просто высшего, но качественного высшего образования.

Своеобразным ответом на эти социальные вызовы стало появление образовательных платформ, деятельность которых, в свою очередь, стала источником других глобальных проблем тоже этического свойства. Эти агрегаторы дистанционных курсов предоставляют массовые открытые онлайн-курсы (МОOК - от англоязычной аббревиатуры МООС - massive open online courses) от ведущих университетов мира. Это полноценные учебные курсы и целые блоки курсов по отдельным специальностям с видеолекциями, конспектами, домашними заданиями и экзаменами. В итоге, студенты, успешно освоившие тот или иной курс, получают сертификаты.

К числу наиболее известных образовательных платформ относятся Coursera, EdX, Udemy, Canvas, FutureLearn, Udacity, Khan Academy, китайская Squirrel AI learning и мн. др., на сегодняшний день имеющие уже десятки миллионов пользователей (слушателей) по всему миру.

По данным на 2017 г. университетские онлайн-курсы слушали 80 млн чел. Крупнейшие образовательные платформы имеют уже десятки миллионов пользователей по всему миру: Coursera - 30 млн, edX - 14 млн, FutureLearn - 7 млн, Udacity -5 млн чел. [2, с. 7]. В одном только Китае, представляющем собой крупнейший мировой рынок цифрового образования, 142 млн чел. обучаются онлайн (данные на 2019 г.; правда, статистика в это число включает не только студентов, но и школьников) [3]. 
Эксперты полагают, что через пять лет мировая аудитория онлайн-курсов составит около 200 млн чел. [2, с. 7]

Глобальные онлайн-платформы небезосновательно рассматриваются как реальный конкурент «классического» очного образования. Это прототип глобальной системы, которая со временем, по мере развития спутникового Интернета, позволит получать достойное образование жителям даже самых отдаленных регионов. Не слу чайно, уже сейчас Coursera позиционируется как «лучшее в мире обучение, доступное во всех уголках земного шара» [4] (В версии Ш. Янга: «Мы создаем мир, в котором каждый человек в любой точке мира сможет изменить свою жизнь с помощью лучшего в мире образования» $[5$, с. 26]).

Естественно, что подобная мировая экспансия дистанционного образования, оттягивающего на себя слушателей и финансовые ресурсы, является потенциальной угрозой существования традиционных университетов, способной оставить без работы десятки тысяч преподавателей высшей школы. Существует немало исследований, согласно которым те образовательные организации, которые уже сейчас не внедряют различные формы дистанционного обучения, просто не выживут в конкурентной борьбе за потенциальных клиентов [1, с. 69].

И это главная, весьма тревожащая этическая проблема и уже видимый результат внедряемого ныне дистанционного образования, хотя прогноз основателя Udacity Себастьяна Труна о том, что через 50 лет останется всего 10 высших учебных заведений (и все - онлайн) $[5$, c. 23$]$ вряд ли осушествится в полной мepe.

Уже сейчас футурологи называют профессию преподавателя находящейся под угрозой. Ясно, что традиционная система образования должна будет измениться, стать более пластичной, динамичной, отвечающей на вызовы времени.
Понятно, что аудиторная работа, когда преподаватель оперировал только конспектом лекции и, в лучшем случае, располагал доской и мелом, безвозвратно ушла в прошлое. Сам преподаватель должен меняться, соответствовать духу компьютерной эпохи.

Эксперты в сфере образования утверждают, что на сегодняшний день онлайн и офлайн-обучение уже примерно равноценны по качеству и конечному результату [6]. Но и здесь тоже есть «подводные камни». Оказывается, что для недостаточно мотивированного студента, лишенного возможности общаться с «живым» преподавателем, результаты будут хуже, чем если бы он слушал очные курсы в университете. И еще один негативный аспект - старшие школьники и студенты в ситуации исключительно дистанционного образования оказываются в большей психологической зависимости от своих гаджетов, что негативно влияет на успешность их дальнейшей социализации и возможность работы в коллективе.

Ещё более неоднозначно обстоит ситуация с младшими школьниками. В ситуации, когда с ними будет общаться электронный тьютор с элементами искусственного интеллекта, есть опасение, что у обучающихся младшего возраста может поменяться восприятие «живого», что, в свою очередь, помешает развитию эмоционального интеллекта $[1$, с. 75$]$.

В то же время, оценивая перспективы и угрозы новых (дистанционных) технологий для «традиционного» обучения, такой авторитет в сфере образования, как В.А. Садовничий заявляет, что, вероятно, еще долго живое общение будет полноценнее дистанционного. К тому же, конкретные цифры показывают, что получение полноценного высшего образования в интернете с получением диплома еще не стало общим трендом. В 2017 г. таковых на онлайн-ресурсах было лишь 7 тыс. чел. [2, с. 7]. Главным образом 
интернет-платформы используются для переобучения и специализации, и не случайно, что $89 \%$ пользователей платформы Coursera - люди старше студенческого возраста $[2$, с. 7$]$.

Однако ясно, что доля «дистанта» с годами будет только возрастать; одновременно будут становиться более актуальными новые проблемы, связанные с освоением человечеством цифровых технологий.

Целый ряд новых этических проблем порождается различными трендами, обозначившимися в дистанционном образовании в последние годы. Сегодня наиболее значимыми из них являются персонализация и адаптивный подход, предиктивная аналитика [3].

Персонализация и адаптивный подход при использовании дистанционных технологий позволяют проанализировать психологические особенности обучаемого и предложить учебный материал в том формате и того уровня сложности, который оптимально подходит ему. «При адаптированном обучении образовательный контент перестает быть фиксированным объемом знаний, он становится динамичным, меняется в зависимости от актуального статуса ученика или студента, - отмечает Ивонн Ляо, партнер Squirrel AI learning - крупнейшей китайской EdTech-компании, развивающей технологии адаптивного обучения на основе алгоритмов искусственного интеллекта. - Система адаптируется к его потребности и скорости освоения материала. Посредством мониторинга продвижения студента в реальном времени удается создать индивидуальный дизайн его образовательной траектории» [7]. Это не только в разы повышает качество образования, но и снижает риск дискриминации одаренных студентов, поскольку при «традиционном» обучении преподаватели вынуждены ориентироваться на «средний» уровень знаний в группах [3].
Предиктивная (или предсказательная) аналитика в дистанционном образовании связана как с созданием персонализированных образовательных траекторий, так и с рекомендациями о том, какая специализация и карьера больше подойдут тому ли иному человеку. Однако долгосрочные прогнозы, как и любые рекомендации по выбору профессии, сопряжены с определенными этическими проблемами, поскольку алгоритмы и цифровые технологии могут подталкивать пользователя к определенному поведению, ограничивая его свободу воли и вынуждая следовать указаниям аналитической системы.

Кроме того, в целях персонализации и предиктивной аналитики могут собираться данные обо всех достижениях студента. И здесь уже возникает этическая проблема несанкционированного использования персональных данных в сфере профессионального образования или проблема «цифрового следа», когда выпускник вуза может получить отказ от потенциального работодателя, в силу утечки сведений личного характера о его достижениях и неудачах в годы учебы. А ведь некоторые владельцы дистанционных образовательных курсов с массовым интерактивным участием заявляют о готовности продавать сведения о лицах, зачисленных на курс, и рассматривают эту практику как способ получения дополнительного дохода.

Многие их этих проблем приобрели дополнительную остроту в период пандемии COVID-19, вызвавшей необходимость кардинальных преобразований в системе образования (и в том числе, безальтернативный и ускоренный переход на дистанционные формы обучения) $[8$, c. $179-180]$.

Исследователи отмечают, что наиболее уязвимыми в этой ситуации оказались учащиеся из семей с низким материальным статусом, дети мигрантов, иностранные студенты, а также 
учащиеся с ограниченными возможностями здоровья, т.е. именно те группы, для выравнивания возможностей которых в получении образования, собственно, и разрабатывалась сама идея дистанционного обучения. Распространенный характер приобрела ситуация, когда отсутствие соответствующих гаджетов и/или отсутствие доступа к интернету, плохое знание языка преподавания практически парализовало возможности и желание школьников и студентов обучаться дистанционно. Особенно катастрофический характер приобрели эти препоны в развивающихся странах, когда образовательный процесс фактически блокировался ввиду отсутствия компьютеров, интернета и даже электричества, особенно в сельских районах. Еще одним фактором, который следует упомянуть, стало прекращение материальной поддержки малообеспеченных семей, например, в виде бесплатного школьного питания $[8$, с. $185 ; 9 ; 10 ; 12]$. Всё это говорит о том, что общество оказалось не готово к столь радикальным переменам в системе образования.

Еще одним последствием пандемии, имеющим «отложенный эффект», является ухудшение стартовых возможностей школьников и студентов, завершение обучения которых пришлось на период карантина и которые были лишены возможности полноценного экзаменационного процесса и преддипломной практики.

Есть немало публикаций, в которых преподаватели сетуют на сложность контроля за работой обучающихся в дистанционном формате, который предоставляет для нерадивых студентов больше возможностей для обмана в ходе образовательного процесса [11].

Согласно опросам преподавателей МГТУ, они также столкнулись с данной ситуацией в первоначальный период перехода на дистант, когда с весны 2020 г. пришлось перенести учебный процесс в ЭИОС. Например, ситуация, когда студент должен был письменно выполнить задание семинарского занятия и выслать его преподавателю. Данная форма работы вызвала настоящую эпидемию подлогов, когда от целой группы преподаватель получал абсолютно идентичные тексты. Причем сами студенты объясняли неизбежность этого нехваткой времени, поскольку, по их словам, объем домашних заданий при дистанционке увеличился в несколько раз. Следует отметить, что позднее, при работе в системах ZOOM и Mind, когда преподаватели на семинарах получили возможность прямого визуального контроля, подобная проблема отпала сама собой.

Таким образом, дистанционный формат образования, несмотря на свои колоссальные потенциальные возможности, имеет целый ряд скрытых проблем, которые обострились с началом пандемии. Дальнейшее развитие онлайн-образования невозможно без учета факторов морально-этического плана.

Опыт работы отечественной и мировой системы образования в условиях пандемии требует глубокого изучения и обобщения для корректирования методов и темпов инновационных процессов в современной педагогике, а также для уточнения места, характера и значимости дистанционного обучения в образовательной системе.

\section{ЛИТЕРАТУРА:}

1. Шишкова А.В. Цифровая этика в современном образовательном процессе // Социальногуманитарные знания. 2019. № 2. С. 68-76.

2. Садовничий В.А. Университеты, общество и будущее человечества // Вестник Московского университета. Серия, 20: Педагогическое образование. 2019. № 2. С. 3-20. 
3. Этика и цифра: этические проблемы цифровых технологий [Электронный ресурс]: аналитический доклад / РАНХиГС; Центр подготовки руководителей цифровой трансформации. URL: https://ethics.cdto.ranepa.ru/ (дата обращения: 16.05.2021).

4. https://www.coursera.org/ (дата обращения: 20.03.2021).

5. Янг Ш. От подрыва к инновациям: о будущем МООК // Вопросы образования. 2018. № 4. C. 21-43.

6. Онлайн-курсы не менее эффективны, чем офлайн-форматы [Электронный ресурс] / НИУ «Высшая школа экономики». URL: https://iq.hse.ru/news/217043836.html. (дата обращения: 06.04.2021).

7. Любимцева Ю. Учитель в эпоху технологического прорыва [Электронный ресурс] // EdExpert. URL: http://edexpert.ru/technological-breakthrough. (дата обращения: 05.04.2021).

8. Радина Н.К., Балакина Ю.В. Вызовы образованию в условиях пандемии: обзор исследований // Вопросы образования. 2021. № 1. С. 178-194.

9. Омельченко Е.'А. Образование детей из семей международных мигрантов в условиях пандемии // Этнодиалоги. 2020. № 3 (61). С. 54-64.

10. Плетнева Ю.Э., Очирова Г.Н. Влияние пандемии COVID-19 на положение иностранных студентов в России // Научное обозрение. Серия, 1: Экономика и право. 2020. № 3. С. 147-155.

11. Об этическом аспекте дистанционного обучения. Интервью с преподавателем НГТУ Батаевым [Электронный ресурc]. URL: https://zen.yandex.ru/media/4sinfo/ob-eticheskom-aspektedistancionnogo-obucheniia-interviu-s-prepodavatelem-ngtu-bataevym-5f05a22937d2111la67d0e5a (дата обращения: 10.05.2021).

12. Oyedotun TiD. Sudden Change of Pedagogy in Education Driven by COVID19: Perspectives and Evaluation from a Developing Country // Research in Globalization. 2020. Vol. 2.

\section{REFERENCES:}

1. Shishkova A.V. Digital ethics in the modern educational process // Social and humanitarian knowledge. 2019. No. 2. P. 68-76.

2. Sadovnichy V.A. Universities, society and the future of mankind // Bulletin of Moscow University. Series, 20: Teacher education. 2019. No. 2. P. 3-20.

3. Ethics and number: ethical problems of digital technologies [Electronic resource]: analytical report / RANEPA; Center for training leaders of digital transformation. URL: https://ethics.cdto. ranepa.ru/ (date of access: 16.05.2021).

4. https://www.coursera.org/ (date of access: 20/03/2021).

5. Young Sh. From disruption to innovation: on the future of MOOCs // Education Issues. 2018. No. 4. P. $21-43$.

6. Online courses are no less effective than offline formats [Electronic resource] / NRU "Higher School of Economics". URL: https://iq.hse.ru/news/217043836.html. (date of access: 06/04/2021).

7. Lyubimtseva Yu. Teacher in the era of technological breakthrough [Electronic resource] // EdExpert. URL: http://edexpert.ru/technological-breakthrough. (date of access: 05.04.2021).

8. Radina N.K., Balakina Yu.V. Challenges to education in the pandemic: a survey of research // Education Issues. 2021. No. 1. P. 178-194.

9. Omelchenko E.A. Education of children from families of international migrants in a pandemic // Ethnodialogi. 2020. No. 3 (61). P. 54-64.

10. Pletneva Yu.E., Ochirova G.N. The impact of the COVID-19 pandemic on the situation of foreign students in Russia // Scientific Review. Series, 1: Economics and Law. 2020. No. 3. P. $147-155$. 
11. On the ethical aspect of distance learning. Interview with Batayev-NSTU teacher [Electronic resource]. URL: https://zen.yandex.ru/media/4sinfo/ob-eticheskom-aspekte-distancionnogo-obucheniiainterviu-s-prepodavatelem-ngtu-bataevym-5f05a22937d2111la67d0e5a (date of access: 10/05/2021).

12. Oyedotun T.D. Sudden Change of Pedagogy in Education Driven by COVID 19: Perspectives and Evaluation from a Developing Country // Research in Globalization. 2020. Vol. 2. 